Article original

\title{
Facteurs prédictifs de rémission partielle dans le diabète de type 1: à propos d'une cohorte de 117 enfants et adolescents
}

\author{
Predictive factors of partial remission in 117 children and adolescents with type \\ 1 diabetes
}

Amel ZENNAKI' , Malika NACEUR', Charef LATROCH' , Asma AOUI' , Mounia GHARNOUTR', Nesma TALEB

Ourida', Ghazalia BOUDRAA', Karim BOUZIANE-NEDJADI', Mahmoud TOUHAMI'

${ }^{1}$ Service de pédiatrie «C» (Clinique A. Cabral) CHU d'Oran, Algérie

¿service de pédiatrie, Hôpital Militaire Régional d’Oran, Algérie

Auteur correspondant: a.zennaki@yahoo.fr

Article reçu le 5/10/2018/Accepté le 17/12/2018

\section{MOTS CLÉS \\ Diabète de type 1 , rémission partielle, lune de miel, enfant, HbA1c.}

\section{Résumé}

Introduction / objectifs - Dans le diabète de type 1, la rémission partielle a un effet bénéfique sur les complications aigues et chroniques. Notre objectif était de décrire la rémission partielle et d'identifier ses facteurs prédictifs dans une cohorte d'enfants et d'adolescents atteints de cette maladie.

Patients et méthodes - Les enfants et adolescents âgés de moins de 15 ans, admis entre juin 2013 et juillet 2014, ayant débuté l'insulinothérapie moins d'un mois avant leur admission ont été inclus dans l'étude.

Résultats - Cent-dix-sept (117) nouveaux cas ont été étudiés avec un sex-ratio de 0,86 , ont été analysés. A l'admission, l'âge moyen était de $8,2 \pm 4,3$ ans. L'acidocétose inaugurale était présente dans $26 \%$ des cas. La rémission (moins de $0,5 \mathrm{U} /$ $\mathrm{Kg} / \mathrm{j}$ d'insuline et $\mathrm{HbA} 1 \mathrm{c} \leq \mathrm{7,5 \%}$ ) a été obtenue chez 21 enfants, soit dans 17,95\% des cas avec une durée moyenne de 5,2 $\pm 4,2$ mois (min. 3-max. 15). La rémission était significativement plus fréquente chez les enfants qui n'avaient pas présenté d'acidocétose inaugurale $(p<0,01)$, chez ceux qui n'avaient pas de germains atteints de DT1 $(p<0,05)$ et chez les filles $(p<0,02)$. Il n'y avait pas de relation significative avec le taux d'HbA1c à l'admission, le niveau d'instruction ou d'occupation maternelle, l'origine géographique et l'âge.

Conclusions - L'acidocétose au diagnostic est un facteur prédictif négatif de rémission partielle. L'absence de DT1 chez les germains et le sexe féminin sont des facteurs prédictifs de rémission propres à notre contexte de travail. 


\section{KEY WORDS}

Type 1 diabetes, partial remission, honey moon, child, HbA1c.

\section{Introduction}

Dans le diabète de type 1 (DT1), la rémission partielle, ou «lune de miel», peut être définie comme la période qui suit de quelques jours à quelques semaines la mise en route d'un traitement par l'insuline, lorsque celui-ci nécessite moins de $0,5 \mathrm{U} / \mathrm{Kg} / 24$ heures d'insuline et que l'HbA1c se maintient au-dessous de $7 \%$ (1-3) ou de $8 \%$ pour d'autres (4). Elle peut durer des semaines ou des mois, permettant un meilleur départ dans la maladie du fait d'un équilibre glycémique plus facile à obtenir grâce à la persistance d'une insulino-sécrétion résiduelle notable, de la diminution des appréhensions maternelles quant aux grandes variations glycémiques et d'une meilleure écoute lors des séances de formation thérapeutique. Elle ne doit pas signifier pour autant une rémission totale du diabète, faux espoir, qu'il convient de ne pas entretenir chez les parents (2). Les résultats du Diabetes Control and Complications Trial (DCCT) montrent que la préservation de la sécrétion résiduelle était non seulement associée avec une réduction des complications micro- et macro vasculaires à long terme, mais aussi la réduction des complications aiguës (5-9).

Une nouvelle définition de la période de rémission a été proposée $(9,10)$. Elle comprend à la fois le contrôle glycémique et la dose d'insuline. C'est l'HbA1c ajustée à la dose d'insuline: insulin-dose-adjusted HbA1c (IDAA1C) $=\mathrm{HbA} 1 \mathrm{C}(\%)+$
4 (dose d'insuline $(\mathrm{Ul} / \mathrm{kg} / \mathrm{j}))$. Une valeur $\leq 9 \%$ correspond à la rémission partielle. Cette nouvelle définition reflète la fonction résiduelle $B$-cellulaire et aurait une meilleure stabilité par rapport aux définitions conventionnelles.

Les facteurs influençant la phase de rémission sont nombreux (11). Différentes études ont montré des résultats qui peuvent sembler contradictoires. La préservation de la masse cellulaire résiduelle $B$ a pu être également obtenue par des immunosuppresseurs (12) ou la vitamine E (13) entraînant ainsi des rémissions partielles plus ou moins prolongées.

Il est important de souligner que l'on peut agir sur certains des facteurs délétères influençant négativement la rémission comme l'acidocétose par le biais d'une sensibilisation et d'une intervention précoces (14-16), ce qui aura pour effet un bon départ dans la maladie avec une réduction des complications aigues et chroniques. Il est également intéressant de noter que d'autres facteurs ont été décrits comme ayant une influence sur la rémission partielle comme le régime sans gluten $(17,18)$. Aucune étude sur la rémission chez l'enfant et l'adolescent n'a été faite en Algérie. L'objectif de notre travail était de déterminer le taux de rémission partielle et d'identifier ses facteurs prédictifs dans une cohorte d'enfants et d'adolescents atteints de DT1 durant les 15 premiers mois de la maladie. 


\section{Patients et méthodes}

\section{Patients}

Ont été retenus, les nouveaux cas de DT1 de l'enfant admis consécutivement entre juin 2013 et juillet 2014, âgés de moins de 15 ans, soit 117 enfants et adolescents, ayant débuté leur insulinothérapie à leur admission dans le service, ou dans le mois, enseignés et suivis un minimum d'une année dans le service.

Ont été exclus les diabètes autre que DT1 (DT induit, DT2, DT monogénique), enfants traités par insuline depuis plus d'un mois avant leur admission dans le service.

\section{Méthodes}

Ce travail est une étude prospective menée au service de pédiatrie $C$ du 1er juin 2013 au 1er juillet 2014. Les enfants et leurs mères ont été pris en charge en hospitalisation de jour. L'insulinothérapie a été débutée ou poursuivie, et l'éducation thérapeutique a été faite en parallèle.

Durant l'hospitalisation de jour, les patients et leurs mères sont retenus de $8 \mathrm{~h} 30$ à $16 \mathrm{~h}$ en semaine et de $9 \mathrm{~h}$ à $12 \mathrm{~h}$ le week-end. En dehors des heures de présence dans le service, un contact téléphonique avec l'équipe soignante était possible 24 heures sur 24.

L'insulinothérapie a consisté, soit en schéma conventionnel optimisé pour les patients ( 3 injections par jour : mélange dans la seringue d'insuline rapide et intermédiaire matin et soir, insuline rapide à midi), soit par schéma basal/bolus aux analogues de l'insuline, soit sous forme d'insulines pré-mélangées avec injection d'insuline rapide à midi.

L'hospitalisation de jour a duré jusqu'à obtention d'un équilibre glycémique satisfaisant (avec la majorité des glycémies dans l'objectif) et/ou jusqu'à l'achèvement fin de l'éducation thérapeutique initiale. Les enfants ont ensuite été revus, après leur sortie administrative, dans les 10 jours en consultation spécialisée, puis après un mois, puis trimestriellement, avec la possibilité de se présenter à tout moment dans les intervalles des rendez-vous programmés et la possibilité de consulter l'éducatrice ou la psychologue téléphoniquement.

Les données relevant de l'hospitalisation initiale ont été recueillies dès l'admission par la même personne, sur une fiche d'enquête préétablie. Les données relevant du suivi, ont été prises de façon rétrospective sur dossier. Ont été recueillies, les données démographiques et socio-économiques des familles, les antécédents médicaux familiaux et les an- técédents de DT1, les circonstances du diagnostic, le mode de présentation du DT1 (acidocétose (ACD), cétose, hyperglycémie), les données cliniques et biologiques au diagnostic et à l'admission, les résultats de la prise en charge initiale en termes de délai d'obtention d'un équilibre satisfaisant et de durée d'hospitalisation. Pour le suivi des patients, ont été recueillis à 3, 6, 9, 12 et 15 mois, l'HbA1c, le schéma insulinique en cours, ainsi que la dose quotidienne d'insuline. L'équilibre glycémique a été évalué par la mesure du taux d'HbA1c dont le dosage était centralisé dans le service sur un automate de marque Drew (USA), utilisant la technique de chromatographie à basse pression, échangeuse de cations conjointement à un gradient d'élution. Les valeurs normales de la mesure pour le fabricant sont de 4 à $6 \%$ de l'hémoglobine totale.

Le diagnostic d'ACD a été fait sur des critères cliniques, par défaut de gaz du sang en routine: signes cliniques d'ACD (obnubilation, déshydratation, fièvre, polypnée, nausées, vomissements, douleurs abdominales, crampes musculaires), réhydratation, hospitalisation initiale en service de réanimation. La rémission a été définie, par la diminution des besoins en insuline à moins de $0,5 \mathrm{UI} / \mathrm{kg} / \mathrm{j}$, avec une $\mathrm{HbA} 1 \mathrm{c} \leq 7 \%(3)$. Les paramètres étudiés comme facteurs prédictifs de rémission partielle étaient: l'ACD au diagnostic, le sexe, l'âge, la présence de DT1 chez les germains, l'HbA1c au diagnostic, la Wilaya d'origine et le niveau d'instruction des mères.

Les analyses ont été effectuées avec le logiciel Excel, et le seuil de $5 \%$ a été choisi comme limite de signification. La comparaison d'effectifs a été faite par le test du chi-deux. Tous les patients ayant participé à cette étude ont donné leur consentement. Les investigateurs se sont engagés à ce que cette étude soit réalisée en conformité avec la législation en vigueur du Code de la Santé Publique ainsi qu'en accord avec les Bonnes Pratiques Cliniques. La section de pédiatrie du CHU/Faculté de Médecine d'Oran a donné son accord pour la réalisation de l'étude, à défaut d'un comité de protection des personnes inexistant ou inopérant au niveau d'Oran.

\section{Résultats}

Cent dix-sept nouveaux patients consécutifs atteints de DT1 ont été analysés. Les caractéristiques de la population étudiée sont détaillées dans le Tableau 1. L'âge moyen des enfants était de $8,2 \pm 4,3$ ans. Les enfants âgés de 10 à 14 ans représentaient $37 \%$ des patients. L'IMC se situait au percentile 50 , sans différence notable entre les sexes. Les âges moyens des mères et des pères étaient de $39 \pm 6,9$ et de $47 \pm$ 8,5 ans respectivement. 
La rémission a été obtenue chez 21 patients, soit dans 17,95 $\%$ des cas avec une durée moyenne (entre le début et la fin de la rémission) de 5,2 $\pm 4,2$ mois. La durée médiane était de 3 mois. Les facteurs prédictifs de rémission sont représentés dans le Tableau 2.
Les enfants qui n'avaient pas d'ACD au diagnostic, ceux n'ayant pas de germains atteints de DT1 et les filles obtenaient significativement plus de rémissions. Il n'y avait pas de relation significative avec le taux d'HbA1c à l'admission, le niveau d'instruction ou d'occupation maternelle, ou les classes d'âge.

Tableau 1. Caractéristiques de la population étudiée à l'admission

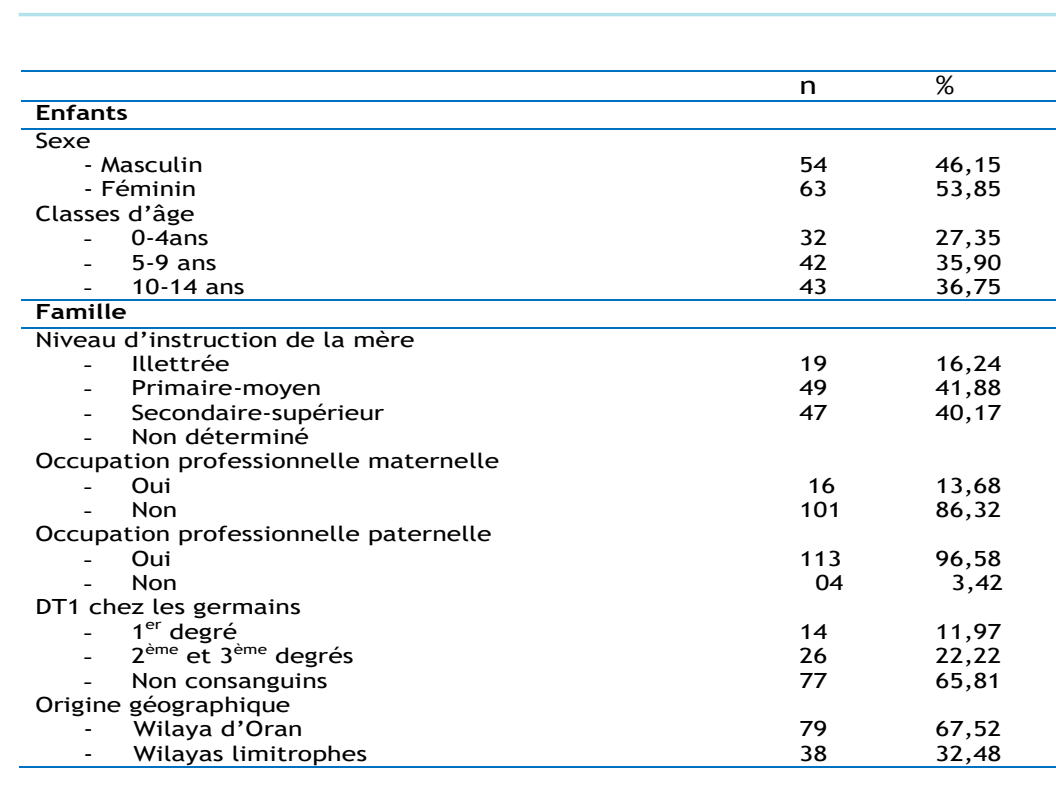

Tableau 2. Facteurs prédictifs de rémission partielle

\begin{tabular}{|c|c|c|c|}
\hline \multirow{2}{*}{ Facteur prédictif } & \multicolumn{2}{|c|}{ Rémission partielle } & \multirow[b]{2}{*}{$p$} \\
\hline & Rp/n exposés & & \\
\hline $\begin{array}{l}\text { Acidocétose } \\
\text { Présence d'ACD } \\
\text { Absence d'ACD }\end{array}$ & $\begin{array}{r}2 / 40 \\
19 / 77\end{array}$ & $\begin{array}{r}5,00 \\
24,68\end{array}$ & $\begin{array}{l}p< \\
0,0 \\
1\end{array}$ \\
\hline \multicolumn{4}{|c|}{$\begin{array}{l}\text { Présence de germains DT1 } \\
\text { Présence de germains }\end{array}$} \\
\hline $\begin{array}{l}\text { Absence de germains } \\
\text { (1er et 2ème degré) } \\
\text { Sexe }\end{array}$ & $17 / 77$ & 22,08 & $\begin{array}{l}p< \\
0,0 \\
5\end{array}$ \\
\hline Filles & $15 / 63$ & 23,31 & \\
\hline Garçons & $6 / 54$ & 11,11 & $\begin{array}{l}p< \\
0,0 \\
2\end{array}$ \\
\hline \multicolumn{4}{|l|}{ HbA1c à l'admission } \\
\hline$<10 \%$ & $10 / 38$ & 26,32 & \\
\hline$>10 \%$ & $11 / 58$ & 18,96 & $\begin{array}{l}p= \\
0,7 \\
6\end{array}$ \\
\hline \multicolumn{4}{|l|}{ Wilaya d'origine } \\
\hline W. Oran & $7 / 38$ & 18,42 & \\
\hline W. limitrophes & $14 / 79$ & 17,72 & $\begin{array}{l}\mathrm{p}= \\
\mathrm{O}, 9\end{array}$ \\
\hline \multicolumn{4}{|c|}{ Niveau d'instruction des mères } \\
\hline $\begin{array}{l}\text { Illet./prim./moyen } \\
\text { sec./univ. }\end{array}$ & $\begin{array}{l}10 / 70 \\
11 / 47\end{array}$ & $\begin{array}{l}14,29 \\
23,40\end{array}$ & $\begin{array}{l}\mathrm{p}= \\
\mathrm{O}, 1 \\
1\end{array}$ \\
\hline \multicolumn{4}{|c|}{ Travail extérieur de la mère } \\
\hline Travail- & 20/101 & 19,80 & $\begin{array}{l}\mathrm{p}= \\
0,2 \\
4\end{array}$ \\
\hline
\end{tabular}




\section{Discussion}

Cette étude a été effectuée au service de Pédiatrie $C$ (clinique A. Cabral) du CHU d'Oran. Le recrutement du service a pratiquement doublé depuis le début des années 2000, avec un pourcentage constant de $44 \%$ de patients en provenance des wilayates (départements) limitrophes de celle d'Oran $(19,20)$. Le service de pédiatrie « $C$ » prend en charge entre 200 et 250 nouveaux patients atteints de DT1 par an avec une cohorte régulièrement suivie de 1400 patients environ. Les facteurs influençant la phase de rémission sont nombreux (11). Il a été montré de longue date que l'insulinothérapie intensive sous toutes ses modalités, pancréas artificiel $(21,22)$, infusion à la pompe externe ou injection sous-cutanée $(23,24)$ préserve le peptide- $C$ et favorise un meilleur équilibre glycémique apprécié par l'HbA1c, la baisse des besoins en insuline et la rémission partielle en termes de fréquence et de durée. Cependant certains auteurs (25) ne retrouvaient pas de différence significative, chez 316 enfants de moins de 15 ans, suivis dans un même centre pédiatrique en Suède de 1976 à 2000, dans l'évolution de la fréquence ou de la durée de la rémission partielle en faisant des comparaisons par périodes de 5 ans et ce malgré des régimes d'insulinothérapie de plus en plus intensifs.

Les facteurs prédictifs d'une rémission partielle sont représentés par le taux élevé de peptide-C (26) et une $\mathrm{HbA} 1 \mathrm{C}$ basse (27). A l'inverse, les facteurs liés à une rémission de mauvaise qualité ou quasiment absente sont la décompensation métabolique inaugurale $(28,29)$, a fortiori, l'acidocétose (30), l'âge inférieur à 5 ans, l'adolescence et la puberté $(1,4,9,29-34)$. Nos résultats montrent un taux faible de rémission comparativement à certains auteurs (35). Ils sont en accord avec d'autres (36) qui retrouvaient des chiffres similaires. La grande variabilité dans les pourcentages de rémission retrouvés entre les études pourrait s'expliquer en grande partie par la variabilité des définitions de la rémission utilisées et par l'âge différent des patients des différentes séries. Le faible taux de rémission dans notre série pourrait être dû en partie au rajeunissement de l'âge au diabète. En effet, $63 \%$ de nos patients avaient moins de 10 ans au diagnostic. Plusieurs études retrouvent une corrélation entre l'âge des patients et la rémission $(8,30,35,36)$.

Dans notre travail la classe d'âge ne semble pas être un facteur prédictif de rémission comme c'est le cas pour de nombreuses études $(1,4,9,30,32,34,35)$. Ceci pourrait s'expliquer, pour les plus jeunes, par la corrélation entre l'âge inférieur à 5 ans et l'acidocétose dans ces études alors que nous ne retrouvions pas de taux plus élevés d'acidocétose inaugurale chez les moins de 5 ans dans notre série. Pour ce qui est des adolescents, les études qui retrouvaient des différences concernaient des adolescents de plus de 12ans (30) alors que nous n'avons pas analysé cette tranche d'âge sé- parément. Nos résultats confirment la corrélation négative entre rémission et acidocétose inaugurale $(3,9,30,35,36)$. Ils ne montrent cependant pas de relation entre taux d'HbA1c au diagnostic et rémission partielle constaté par certains $(26,28)$. Enfin, la prédominance féminine et chez les germains de patients atteints de DT1 semble propre à notre contexte.

\section{Conclusions}

L'absence ACD inaugurale, l'absence de DT1 chez les germains et le sexe féminin sont des facteurs prédictifs de rémission dans notre contexte de travail. Il n'y avait pas de corrélation, dans notre série, avec le taux d'HbA1c à l'admission, le niveau d'instruction maternelle, l'occupation professionnelle ou l'âge de début de la maladie. Nos résultats nous encouragent à mettre encore plus l'accent sur l'intérêt de sensibiliser les familles aux signes du DT1 pour éviter les ACD inaugurales.

\section{Conflits d'intérêt}

Les auteurs déclarent n'avoir aucun conflit d'intérêt.

\section{Références bibliographiques}

[1]. Lombardo F, Valenzise M, Wasniewska M, Messina MF, Ruggeri C, Arrigo T, et al. Two-year prospective evaluation of the factors affecting honeymoon frequency and duration in children with insulin dependent diabetes mellitus: the key-role of age at diagnosis. Diabetes Nutr Metab. août 2002;15(4):246-51.

[2]. Couper J, Donaghue KC. Phases of diabetes in children and adolescents. Pediatr Diabetes. 2009;10(Suppl 12):13-6.

[3]. Couper JJ, Haller MJ, Greenbaum CJ, Ziegler A-G, Wherrett DK, Knip M, et al. ISPAD Clinical Practice Consensus Guidelines 2018 Stages of type 1 diabetes in children and adolescents. Pediatr Diabetes [Internet]. 26 juill 2018 [cité 10 sept 2018]; Disponible sur: https: //onlinelibrary.wiley.com/doi/abs/10.1111/pedi.12734

[4]. Chase HP, MacKenzie TA, Burdick J, Fiallo-Scharer R, Walravens $P$, Klingensmith $G$, et al. Redefining the clinical remission period in children with type 1 diabetes. Pediatr Diabetes. mars 2004;5(1):16-9. [5]. The Diabetes Control and Complications Trial Research Group. The absence of a glycemic threshold for the development of long-term complications: the perspective of the Diabetes Control and Complications Trial. Diabetes. oct 1996;45(10):1289-98.

[6]. The Diabetes Control and Complications Trial Research Group. Hypoglycemia in the Diabetes Control and Complications Trial. The Diabetes Control and Complications Trial Research Group. Diabetes. févr 1997;46(2):271-86.

[7]. Max Andersen MLC, Hougaard P, Pörksen S, Nielsen LB, Fredheim 
S, Svensson J, et al. Partial remission definition: validation based on the insulin dose-adjusted HbA1c (IDAA1C) in 129 Danish children with new-onset type 1 diabetes. Pediatr Diabetes. nov 2014;15(7):469-76. [8]. Max Andersen ML, Nielsen LB, Svensson J, Pörksen S, Hougaard P, Beam C, et al. Disease progression among 446 children with newly diagnosed type 1 diabetes located in Scandinavia, Europe, and North America during the last 27yr. Pediatr Diabetes. août 2014;15(5):345-54.

[9]. Couper JJ, Haller MJ, Ziegler A-G, Knip M, Ludvigsson J, Craig ME, et al. ISPAD Clinical Practice Consensus Guidelines 2014. Phases of type 1 diabetes in children and adolescents. Pediatr Diabetes. sept 2014;15 Suppl 20:18-25.

[10]. Mortensen HB, Hougaard P, Swift P, Hansen L, Holl RW, Hoey $\mathrm{H}$, et al. New Definition for the Partial Remission Period in Children and Adolescents With Type 1 Diabetes. Diabetes Care. août 2009;32(8):1384-90.

[11]. Büyükgebiz A, Cemeroglu AP, Böber E, Mohn A, Chiarelli F. Factors influencing remission phase in children with type 1 diabetes mellitus. J Pediatr Endocrinol Metab JPEM. déc 2001;14(9):1585-96.

[12]. Sobel DO, Henzke A, Abbassi V. Cyclosporin and methotrexate therapy induces remission in type 1 diabetes mellitus. Acta Diabetol. sept 2010;47(3):243-50.

[13]. Crinò A, Schiaffini R, Manfrini S, Mesturino C, Visalli N, Beretta Anguissola $G$, et al. A randomized trial of nicotinamide and vitamin $E$ in children with recent onset type 1 diabetes (IMDIAB IX). Eur J Endocrinol Eur Fed Endocr Soc. mai 2004;150(5):719-24.

[14]. Zennaki A, Ouzzaa A, Gharnouti M, Reguieg A, Niar S, Bouchetara $A$, et al. P115 L'acidose inaugurale dans le DT1: fréquence en pédiatrie, comportement des praticiens devant une suspicion de DT1 et stratégie d'éviction. Diabetes Metab. 2014;40:A56-A57.

[15]. King BR, Howard NJ, Verge CF, Jack MM, Govind N, Jameson K, et al. A diabetes awareness campaign prevents diabetic ketoacidosis in children at their initial presentation with type 1 diabetes. Pediatr Diabetes. déc 2012;13(8):647-51.

[16]. Vanelli M. Education and public information: preventing diabetic ketoacidosis in Italy. Diabetes Voice. 2007;52(special issue):39-41.

[17]. Sildorf SM, Fredheim S, Svensson J, Buschard K. Remission without insulin therapy on gluten-free diet in a 6-year old boy with type 1 diabetes mellitus. BMJ Case Rep. 2012;2012.

[18]. Svensson J, Sildorf SM, Pipper CB, Kyvsgaard JN, Bøjstrup J, Pociot FM, et al. Potential beneficial effects of a gluten-free diet in newly diagnosed children with type 1 diabetes: a pilot study. SpringerPlus. 7 juill 2016;5(1):994.

[19]. Touhami M. Evolution épidémiologique du DT1 de l'enfant à Oran. Implications pour la prise en charge en pédiatrie. In 9èmes journées de diabétologie de l'EHU d'Oran; 2016.

[20]. Niar S. Diabète de type 1 chez l'enfant et l'adolescent : étude du recrutement de l'Ouest algérien. Thèse de doctorat en sciences médicales. Unisersité d'Oran; 2013.

[21]. Mirouze J, Selam JL, Pham TC, Mendoza E. [Remission of diabetes during conventional insulin therapy or therapy controlled by use of an artificial pancreas]. Sem Hôp Organe Fondé Par Assoc Enseign Méd Hôp Paris. 18 févr 1979;55(7-8):354-9.

[22]. Shah SC, Malone JI, Simpson NE. A randomized trial of intensive insulin therapy in newly diagnosed insulin-dependent diabetes mellitus. N Engl J Med. 2 mars 1989;320(9):550-4.

[23]. The Diabetes Control and Complications Trial Research Group. Effect of intensive therapy on residual beta-cell function in patients with type 1 diabetes in the diabetes control and complications trial. A randomized, controlled trial. Ann Intern Med. 1 avr 1998;128(7):517-23.

[24]. de Beaufort CE, Houtzagers CM, Bruining GJ, Aarsen RS, den Boer NC, Grose WF, et al. Continuous subcutaneous insulin infusion (CSII) versus conventional injection therapy in newly diagnosed diabetic children: two-year follow-up of a randomized, prospective trial. Diabet Med J Br Diabet Assoc. déc 1989;6(9):766-71.

[25]. Nordwall M, Ludvigsson J. Clinical manifestations and beta cell function in Swedish diabetic children have remained unchanged during the last 25 years. Diabetes Metab Res Rev. sept 2008;24(6):472-9.

[26]. Zmysłowska A, Młynarski W, Szadkowska A, Bodalski J. [Prediction of clinical remission using the $\mathrm{C}$-peptide level in type 1 diabetes in children]. Pediatr Endocrinol Diabetes Metab. 2007;13(2):71-4.

[27]. Vetter U, Heinze E, Beischer W, Kohne E, Kleihauer E, Teller WM. Haemoglobin A1c: a predictor for the duration of the remission phase in juvenile insulin-dependent diabetic patients. Acta Paediatr Scand. juill 1980;69(4):481-3.

[28]. Vetter U, Heinze E, Kohne E, Teller WM, Kleihauer E. Relation between the degree of initial metabolic decompensation and the duration of the remission phase in type I diabetes mellitus. Eur J Pediatr. févr 1982;138(1):63-6.

[29]. Böber E, Dündar B, Büyükgebiz A. Partial remission phase and metabolic control in type 1 diabetes mellitus in children and adolescents. J Pediatr Endocrinol Metab JPEM. avr 2001;14(4):435-41.

[30]. Bowden SA, Duck MM, Hoffman RP. Young children ( $<5 \mathrm{yr}$ ) and adolescents (>12 yr) with type 1 diabetes mellitus have low rate of partial remission: diabetic ketoacidosis is an important risk factor. Pediatr Diabetes. juin 2008;9(3 Pt 1):197-201.

[31]. Ziegler A-G, Schmid S, Huber D, Hummel M, Bonifacio E. Early infant feeding and risk of developing type 1 diabetes-associated autoantibodies. JAMA. 1 oct 2003;290(13):1721-8.

[32]. Dost A, Herbst A, Kintzel K, Haberland H, Roth CL, Gortner L, et al. Shorter remission period in young versus older children with diabetes mellitus type 1. Exp Clin Endocrinol Diabetes Off J Ger Soc Endocrinol Ger Diabetes Assoc. janv 2007;115(1):33-7.

[33]. Muhammad BJ, Swift PG, Raymond NT, Botha JL. Partial remission phase of diabetes in children younger than age 10 years. Arch Dis Child. avr 1999;80(4):367-9.

[34]. Bonfanti R, Bognetti E, Meschi F, Brunelli A, Riva MC, Pastore MR, et al. Residual beta-cell function and spontaneous clinical remission in type 1 diabetes mellitus: the role of puberty. Acta Diabetol. juill 1998;35(2):91-5.

[35]. Abdul-Rasoul M, Habib H, Al-Khouly M. « The honeymoon phase " in children with type 1 diabetes mellitus: frequency, duration, and influential factors. Pediatr Diabetes. avr 2006;7(2):101-7.

[36]. Bonfanti R, Bazzigaluppi E, Calori G, Riva MC, Viscardi M, Bognetti $\mathrm{E}$, et al. Parameters associated with residual insulin secretion during the first year of disease in children and adolescents with Type 1 diabetes mellitus. Diabet Med J Br Diabet Assoc. oct 1998;15(10):844-50. 